
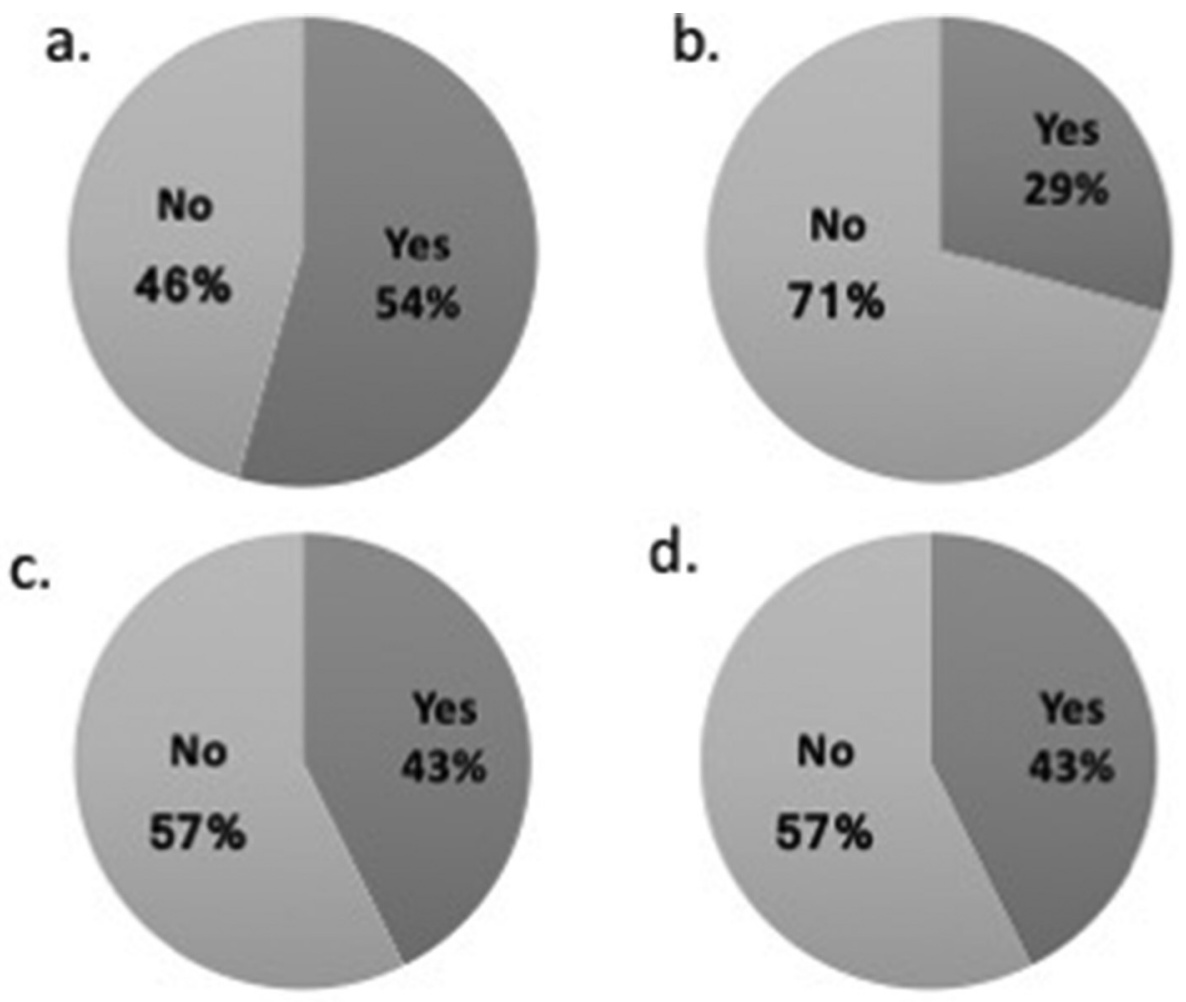

Abstract PS8:155 Figure 2 Individual risk factors for HMB in all menstruating patients. A. passing of large blood clots, B. need for double sanitary protection, C. need for frequent changes of tampons or towels, D. flooding to clothes/bedding

\section{PS8:156 HEMOPHAGOCYTIC SYNDROME IN SYSTEMIC LUPUS ERYTHEMATOSUS. A MONOCENTRIC STUDY OF 20 CASES}

W Ammouri, N Radi, S Toumi, M Bourkia, H Khibri, M Maamar, H Harmouche, M Adnaoui, Z Tazi Mezalek. Internal Medicine Department, Ibn Sina University Hospital, Rabat Morocco, Rabat, Morocco

\subsection{6/lupus-2018-abstract.199}

Objectives We reviewed the medical records of adult patients with SLE for a recent 8 years period and identified patients who had developed HS. The diagnosis of SLE was made using ACR criteria (4 or more criteria) and HS has been diagnosing dusing Hunter criteria (5 or more). We conducted statistical analyses to identify the characteristics of those patients in comparison with SLE patients without HS.

Results Among 208 consecutive lupus patients, 20 patients (19 women) was identified having HS. The mean age of patients was $35.4+11.4$ years (21-68). HS revealed lupus in 7 patients; in the others the delay between diagnosis of SLE and HS was 33 months (1 months - 108). Fever, pericarditis and splenomegaly were found in $95 \%, 70 \%$ and $50 \%$ of patients at presentation of HS. Bone marrow aspiration indicated hemophagocytosis in all patients. Cutaneo-mucous and arthritis were present in $95 \%$ and $70 \%$ of patients at presentation of HS. Bicytopenia or pancytopenia, high C-reactive protein level (mean $74 \mathrm{mg} / \mathrm{L}$ ) and hyperferritinemia (mean $8687 \mathrm{ng} / \mathrm{ml}$ ), hypertriglyceridemia (mean $4.35 \mathrm{~g} / \mathrm{L}$ ) were present in all patients. All patients had anti-nuclear and. Antidouble-stranded DNA antibodies were present in all patients. Serum complement C3 was low in 17 patients.

HS was associated with a lupus flare in 10 patients. Infections was diagnosed in 11 patients.
The most commonly used therapy was corticosteroids, which were initially administered in all patients. Immunosuppressant therapy was used with corticosteroids in 9 patients. Intravenous immunoglobulin was given in 4 cases and Rituximab in one patients. Anti-tuberculosis treatment was the first line treatment in 5 patients. All patients had a good outcome without any mortality with a mean follow-up of 12 months.

Compared with SLE patients without HS, those with HS was significantly older and showed more oral ulcerations, a higher serum C-reactive protein level, a higher ferritinemia, higher SLEDAI and $\mathrm{H}$ score and, splenomegaly.

Conclusion HS was observed in 9,26\% Moroccan patients with SLE. Recognition of the cause of HS was particularly challenging because it may mimic the clinical features of the under lying disease or be confused with an infectious complication.

\section{PS8:157 IDENTIFYING THE RATES OF IRON DEFICIENCY AND ANAEMIA IN PATIENTS WITH SYSTEMIC LUPUS ERYTHEMATOSUS}

C Wincup, A Rahman. Department of Rheumatology, University College London, UK

\subsection{6/lupus-2018-abstract.200}

Background Haematological abnormalities are commonly seen in patients with systemic lupus erythematosus (SLE). Anaemia is frequently reported although there is little data in the literature regarding iron deficiency.

Purpose To identify the prevalence of anaemia and iron deficiency in a large cohort of patients with SLE.

Methods From February 2017, patients fulfilling revised ACR criteria of lupus were prospectively recruited from the Lupus 